at Delft used a linking molecule for the controlled immobilization of the redox enzyme glucose oxidase (GOx), which catalyzes the oxidation of $\beta$-D-glucose to D-glucono-1,5-lactone, on the outer wall of a semiconducting carbon nanotube (CNT) to create a nanosensor that acts both as a reversible $\mathrm{pH}$ sensor and as a sensor capable of measuring GOx activity. CNTs, $600 \mathrm{~nm}$ in length on average, were grown using chemical vapor deposition on degeneratively doped silicon wafers that had a 200-nm thermally grown oxide layer. The researchers conclude from atomic force microscopy images that GOx molecules are immobilized specifically on the SWNTs with a density of about one GOx molecule every $12 \mathrm{~nm}$. Electrodes, composed of a 30-nm gold layer and a 5-nm titanium adhesion layer, were deposited onto the SWNTs using electron-beam lithography. The liquid solution, in which all electrical measurements were made, acts as a very efficient gate for the immersed semiconducting SWNTs.

The researchers show that a substantial decrease in the conductance of SWNTs results from the attachment of only about 50 GOx molecules, thereby demonstrating the potential for sensing the presence of GOx proteins. The researchers said that the decrease in conductance cannot

\section{M5 ${ }^{\text {TM }}$ Plant Update}

Construction of the new, U.S.-based M5TM Pilot Plant in Richmond, Virginia is well underway, and completion is scheduled for early 2004.

Samples for selected applications R\&D will be available after plant commissioning.

For more information on this exciling new fiber technology, please refer to the article on New High Performance Fibers in this issue of MRS Bulletin, or contact us at:

MAGELLAN Systems International, LLC 3 Bethesda Metro Center, Suite 1500-A

Bethesda, MD 20814

240.694 .0007

\section{www.m5tiber.com}

For more information, see http://advertisers.mrs.org simply be due to electrostatic gating by GOx; they propose that it results instead from the decrease in the tube capacitance. They cite numerical estimates of the decrease in conductance, which agree with the experimental measurements, in support of the group's hypothesis.

The conductance of GOx-coated SWNTs is also strongly dependent on $\mathrm{pH}$. The researchers believe that their nanotube sensors are sensitive to and can measure $\mathrm{pH}$ changes with an accuracy of 0.1 $\mathrm{pH}$. They attribute this sensitivity to charged groups on the GOx molecules, which become more negative with increasing $\mathrm{pH}$. This results in a decrease in the electrostatic gate voltage and an increase in the SWNT conductance. In addition, the researchers report that GOxcoated SWNTs also appear to be sensitive to glucose levels. However, quantitative reproducibility of the effect was hampered by substantial low-frequency noise.

The researchers said that higher-mobility devices prepared from short SWNTs $(\sim 20 \mathrm{~nm})$ may provide a powerful tool for enzymatic studies with the ability to measure the activity of even a single redox enzyme. In addition, they look forward to new nanotube biosensors that detect in real time a variety of biomolecules without the need for labeling.

STEVEN TROHALAKI

\section{Biological Template Controls}

\section{Organization of Au Nanoparticles}

Using microorganisms as biological templates is a promising way for assembling nanoparticles into ordered macroscopic structures with architectural control at the nano- , micro-, and macroscopic length scales, but control across multiple length scales is challenging. Different microorganisms such as viruses, bacteria, and fungi can be used as templates for nanoparticle deposition. In the May 23 issue of Angewandte Chemie, a group of researchers led by Chad A. Mirkin at Northwestern University demonstrates how fungi (Aspergillus niger) can be used as templates for assembling gold nanoparticles into highsurface-area semiconducting materials.

Gold nanoparticles of 13-nm diameter were obtained after the reduction of $\mathrm{HAuCl}_{4}(1 \mathrm{mM})$ by trisodium citrate. Prior to assembly, the surfaces of the nanoparticles were modified with alkylthiol-capped oligonucleotide strands; then the modified Au nanoparticles were added to a culture solution consisting of filamentous fungus spores, making a red colloidal solution. During germination, the fungi grow through extension and branching of hyphae (i.e., fine, threadlike filaments). During fungal growth in the culture solu- tion, the microorganisms accumulated gold nanoparticles on the surfaces of the hyphae, turning the red colloid colorless after $\sim 64 \mathrm{~h}$. Air-dried and compressed into thin films, the fungal mats exhibited a golden hue and faint metallic gloss resembling bulk gold. Scanning electron microscopy studies showed that the compacted material preserves the fibrous structure of the Aspergillus niger mycelium. Electrical-transport measurements on this material exhibited Arrhenius-like temperature dependence with an activation energy of $1.62 \mathrm{eV}$.

The structures of single fibers were studied by transmission electron microscopy, revealing that, in vacuum, the hyphae with nanoparticles on the surface collapsed to form thin, nanoparticle-coated, belt-shaped structures. In order to preserve the original structure of the hybrid material, the hyphae coated with nanoparticles were dried in acetone and embedded in an epoxy resin. An ultramicrotomed crosssectional slice of a fungal fiber in epoxy showed a nanoparticle-coated ring structure 3-4 $\mu \mathrm{m}$ in diameter. The researchers reported that a second layer of nanoparticles can be hybridized with the first layer by using nanoparticles with oligonucleotides that are complementary to the first set of particles. This allows the introduction of a secondary structure into the material, they said.

Although the researchers indicate that they do not currently know the nature of the interaction between the nanoparticles and the surfaces of the fungi, they have observed similar phenomena from other common filamentous fungi and actinomycetes. Using these species, the researchers have produced tube-shaped structures up to tens of micrometers long with diameters ranging from $0.8 \mu \mathrm{m}$ to $12 \mu \mathrm{m}$.

MAXIM NIKIFOROV

\section{Influence of Icosahedral Short-Range Order in Undercooled Melts on the Nucleation Barrier Directly Observed}

More than 50 years ago, F.C. Frank hypothesized that undercooled liquids may contain icosahedral short-range order (ISRO), which would be incompatible with the long-range order of the crystalline phase, thus creating an energetic barrier for crystallization. Up to now, however, the hypothesis had not been confirmed by direct experimental observation. K.F. Kelton of Washington University, R.W. Hyers of the University of MassachusettsAmherst, T.J. Rathz of NASA Marshall Space Flight Center, D.S. Robinson of Ames Laboratory, and co-workers have reported in the May 16 issue of Physical 